\title{
Perspectivas dos Graduandos de Enfermagem Frente ao Mercado de Trabalho
}

\author{
Patrícia Novaes Nascimento ${ }^{1}$; Mirella Cristina Leto Barbosa ${ }^{2}$
}

\begin{abstract}
Resumo: O ambiente mercadológico na área da saúde está cada vez mais competitivo, principalmente para o Enfermeiro, que devido a ampliação das graduações sofre com competitividade no mercado, que exige uma boa qualificação e dinamismo para acompanhar o desenvolvimento tecnológico e cientifico que surge. Objetivos: Averiguar em estudos, as perspectivas dos graduandos em Enfermagem sobre o atual mercado de trabalho. Especificamente, objetiva-se caracterizar o perfil dos alunos, analisar o processo de formação acadêmica e expectativas profissionais, e apresentar as principais competências e habilidades para o desenvolvimento profissional. Métodos: O estudo trata-se de uma revisão de literatura, com base numa pesquisa estudo bibliográfica sobre a temática. A busca dos estudos foi realizada em bases de dados do LILACS, Scielo e Google acadêmico. O critério de inclusão foi de pesquisas que abordem as perspectivas dos graduandos em enfermagem quanto ao seu futuro profissional. Resultados: A análise dos estudos permite perceber que as principais demandas dos graduandos quanto à inserção no mercado de trabalho se relacionam com a insegurança e a dificuldade de relacionar o conhecimento teórico com a prática profissional. Além disso, percebe-se que a visão dos graduandos sobre o futuro profissional reflete as oportunidades experimentadas durante a graduação. Conclusão: Diante da necessidade atendimento das demandas da área da saúde, deve-se implementar mudanças curriculares no programa de graduação em Enfermagem nos últimos anos, buscando atingir essa prática diferenciada, para que os futuros Enfermeiros possam desenvolver-se profissionalmente, de modo crítico e reflexivo, atendendo todas as necessidades do mercado de trabalho.
\end{abstract}

PALAVRAS-CHAVE: Enfermagem. Graduação; Mercado de Trabalho

\section{Graduates' Perspectives of the Nursing Labor Market}

\begin{abstract}
The market environment in the health area is becoming more competitive, especially for the Nurse, who due to the graduation increase suffers with competitiveness in the market, which requires a good qualification and dynamism to accompany the technological and scientific development that arises. Objectives: To find out in studies the perspectives of Nursing undergraduates about the current job market. Specifically, it aims to characterize the profile of students, analyze the process of academic training and professional expectations, and present the main skills and abilities for professional development. Methods: The study is a literature review, based on a research study on the subject. The search of the studies was carried out in LILACS, Scielo and Google academic databases. The inclusion criterion was research that addresses the perspectives of nursing undergraduates regarding their professional future. Results: The analysis of the studies allows us to understand that the main demands of the undergraduate students regarding their insertion in the labor market are related to the insecurity and the difficulty of relating theoretical knowledge to professional practice. In addition, it is perceived that the vision of the graduates on the future professional reflects the opportunities experienced during the graduation. Conclusion: Given the need to meet the demands of the health area, curricular changes should be implemented in the undergraduate Nursing program in the last years, seeking to achieve this differentiated practice, so that future Nurses can develop professionally, critically and Reflective of all the needs of the labor market.
\end{abstract}

Keywords: Nursing. University graduate; Job market

\footnotetext{
${ }^{1}$ Graduação em Enfermagem pela Faculdade Independente do Nordeste). Autor correspondente: patynovaes2007@ hotmail.com;

${ }^{2}$ Enfermeira Sanitarista Graduada em Enfermagem e Obstetrícia pela Universidade Estadual do Sudoeste da Bahia. Mestre em Saúde Coletiva com área de concentração em Serviços e Sistemas de Saúde pelo Instituto de Saúde Coletiva / UFBA. Especialista em Saúde Coletiva pelo Instituto de Saúde Coletiva / UFBA e em Educação Profissional na área da saúde: Enfermagem, pela Fundação Oswaldo Cruz. Docente da Faculdade Independente do Nordeste - FAINOR.
} 


\section{Introdução}

O mercado de trabalho está cada vez mais competitivo, atingindo inclusive a área da saúde. Assim, exige-se atualmente uma mão de obra cada vez mais qualificada, onde os profissionais devem ser dinâmicos para acompanhar todo o desenvolvimento tecnológico e cientifico que surge nessa área, se tornando essas demandas grandes desafios principalmente a formação do enfermeiro.

Nesse contexto, diante da necessidade atendimento as necessidades mercadológicas da área da saúde, inúmeras mudanças curriculares ocorreram no programa de graduação em Enfermagem nos últimos anos, buscando atingir essa prática diferenciada. Segundo apontam Oliveira, Nunes e Musse (2016), os desafios que atualmente emergem na formação acadêmica superior em enfermagem estão em construir um profissional, com competências e habilidades para intervir nos serviços de saúde, de forma crítica e reflexiva, aliando de forma qualificada e eficaz a teoria com a prática, além disso, espera-se que o enfermeiro desenvolva sua atividade profissional como um agente integrante de politicas de saúde, promovendo assim um verdadeiro processo de empoderamento dos direitos e deveres dos usuários.

No entanto, ainda se tem grandes lacunas entre a vivência acadêmica e a vivencia profissional na área da enfermagem, pois como salientam Jabbur, Costa e Dias (2012), a realidade vivenciada durante a vida acadêmica difere muito das situações reais nos serviços de saúde onde o profissional vive frente a dilemas, incertezas, conflitos pessoais e falta de recursos para desenvolvimento de seu trabalho.

Apresentando uma mesma percepção Spindola et al (2011), enumeram em seu estudo, que nas faculdades de enfermagem é notória a identificação de jovens ou mesmo adultos, que desconhecem a profissão de enfermagem, suas possibilidades, e suas dificuldades, expressando insegurança quanto ao seu futuro profissional.

Diante dessas concepções e entendendo que é de importância fundamental um processo de reflexão quanto ao processo de formação e os enfrentamentos presentes no cotidiano laboral do Enfermeiro, se torna relevante o conhecimento das perspectivas dos graduandos em enfermagem, possibilitando assim a analise e reflexão sobre as principais demandas que envolvem a formação acadêmica e as características inerentes ao ingressante na profissão. 
Nesta perspectiva, esse estudo teve como questão norteadora: Qual a percepção sobre o mercado de trabalho que o graduando em enfermagem apresenta nos semestres finais? Para responder a este questionamento a pesquisa buscou pesquisar em diversos estudos as perspectivas dos graduandos em enfermagem sobre o atual mercado de trabalho. De forma especifica, objetiva-se nesse estudo a caracterização do perfil dos alunos, a análise do processo de formação acadêmica e expectativas profissionais, e a apresentação das principais competências e habilidades para o desenvolvimento profissional na área da saúde.

A justificativa e relevância deste estudo estão em sua contribuição para a compreensão sobre a enfermagem como profissão, e sua importância dentro dos estabelecimentos, sejam eles de atenção primária, secundária ou terciária, e as percepções que os futuros enfermeiros da graduação apresentam sobre a complexidade e as dificuldades do mercado de trabalho.

Desde a implantação do primeiro curso de enfermagem no Brasil, é possível observar que esses procuravam adequar a sua grade curricular de acordo com o momento vivido pela sociedade. Ocorrendo, assim, mudanças no perfil profissional dos enfermeiros conforme as mudanças no paradigma da saúde e as necessidades do mercado de trabalho de cada época (ITO et al., 2006).

Essas mudanças atualmente estão regidas nas Diretrizes Curriculares Nacionais do Curso de Graduação em Enfermagem (DCN/ENF) implementadas pela resolução n 3/2001 do Conselho Nacional de Educação, que desencadearam um novo movimento de reestruturação de currículos. A principal mudança se baseia na formação de um novo perfil de profissional e de abordagem no processo saúde-doença, voltado, sobretudo, para a realidade da população e de modo coerente com os pressupostos do SUS. Para tanto, surgiram, nas instituições de ensino superior Projetos Políticos Pedagógicos, com a intenção de garantir conteúdos mínimos, flexibilidade na organização do curso, adoção de metodologias ativas, incorporação de atividades complementares, interdisciplinaridade, articulação entre a teoria e a pratica e a pesquisa de extensão (REBOLCAS e FERNANDES, 2013).

\begin{abstract}
As diretrizes curriculares nacionais para a enfermagem orientam a gênese de uma formação generalista, conjeturam prepará-lo para tomar iniciativas, gerir o cuidado, a força de trabalho, os recursos físicos e materiais e de informação. São claras quanto aos papéis do professor e do aluno no processo de ensino e aprendizagem, no qual o aluno é o centro da aprendizagem, cabendo ao professor o permanente desafiar do raciocínio do aluno e pela sua progressiva integração em novos conhecimentos e níveis crescentes de complexidade. Da mesma forma, ensejam um enfermeiro
\end{abstract}


empreendedor e líder na equipe em que atua (KRAIMER, DUARTE E KAISER, 2011, p. 40).

Diante desses novos desafios, a formação em enfermagem tem buscado constituir um profissional, com competências e habilidades para intervir devidamente nos serviços de saúde. Segundo Silva et al (2010), busca-se formar um enfermeiro critico, reflexivo e que consiga aliar a teoria com a pratica sem comprometer os princípios científicos, mas que seja criativo e aprenda a adaptar-se as singularidades e complexidade das situações, além disso espera-se que esse profissional atue como agente integrante de politicas de saúde publica, promovendo o empoderamento dos usuários e participação social.

Fazendo abordagem semelhante Oliveira et al (2014), apontam que:

\begin{abstract}
A Enfermagem, como profissão, busca a formação de um corpo teórico próprio e através de estudos e pesquisas tem a projetado como ciência. As pesquisas e os campos de atuação na enfermagem tem crescido substancialmente nos últimos anos, abrindo perspectivas de conhecimento em múltiplas direções e espaços. Ampliando seu campo de atuação insere perspectivas de conhecimento em múltiplos cenários, incluindo a saúde publica e coletiva, terapias complementares, novas metodologias ativas no ensino, entre outros (p. 93).
\end{abstract}

Dessa forma percebe-se que na contemporaneidade, o mercado de trabalho na área da saúde tem exigido uma mão de obra cada vez mais qualificada, capaz de acompanhar todo o desenvolvimento tecnológico e cientifico da área, tornando-se assim um desafio para a formação do enfermeiro.

Nesse sentido, Oliveira et al (2014) relatam que o mercado de trabalho em saúde, tem realizado mudanças significativas, com a reorganização do mundo do trabalho em saúde e das exigências no perfil de profissionais, promovendo transformações no processo de formação. No entanto, essa realidade requer das instituições formadoras a implementação de ações de reorientação do processo de formação, para que o mesmo promova a aquisição de competências e habilidades para o exercício de práticas e saberes necessárias ao mercado.

$\mathrm{Na}$ literatura, pesquisas mostram alguns exemplos de fragilidades na graduação relacionadas a falta de preparo de enfermeiros para atender as necessidades do exercício da profissão, especialmente no que tange aos relacionamentos interpessoais e gerenciamento de conflitos. Nesse sentido, Jabur, Costa e Dias (2012), apontam que a realidade vivenciada 
durante a vida acadêmica difere muito das situações reais nos serviços de saúde onde dilemas, incertezas, conflitos pessoais e falta de recursos são enfrentados.

Em concordância com esse pensamento, Silva et al (2010), em sua pesquisa, verificaram a existência de várias lacunas existentes entre a trajetória acadêmica e o atual mercado de trabalho da enfermagem, evidenciando a importância que os conhecimentos acerca da realidade do campo profissional representam para a academia

Dessa forma, como afirmam Barlemet al (2012) é fundamental uma reflexão quanto a esse processo de formação, a partir de experiências e enfrentamentos presentes no cotidiano de trabalho, confrontando as competências adquiridas e desenvolvidas no curso de graduação com a realidade laboral. Portanto, conhecer as perspectivas dos graduandos em enfermagem e uma forma de analisar, compreender e refletir sobre as questões relativas ao ensino superior e as características inerentes ao ingressante na profissão.

\section{Método}

O presente estudo trata-se de uma revisão de literatura, com base numa pesquisa estudo bibliográfica sobre a temática. O local de realização da pesquisa foi uma Faculdade privada do interior da Bahia, em Vitória da Conquista/BA. Vitória da Conquista é o terceiro maior município da Bahia, com pouco mais de 340 mil habitantes (IBGE, 2017), estando situado na região sudoeste da Bahia.

Quanto aos aspectos metodológicos, optou-se pela revisão de literatura por permitir através da análise da literatura, o entendimento de um determinado assunto baseando-se em estudos anteriores (LIMA, 2000 apud MENDES, SILVEIRA e GALVÃO, 2008). É um método de pesquisa que tem a finalidade de reunir e resumir parte do conhecimento científico já produzido sobre o tema investigado, ou seja, permite buscar, avaliar e sintetizar as evidências disponíveis para contribuir com o desenvolvimento do conhecimento na temática (MENDES, SILVEIRA e GALVÃO, 2008).

Para a realização dessa revisão foram estabelecidas as seis etapas com base nas recomendações de Mendes, Silveira e Galvão (2008). A primeira etapa consiste na identificação do tema e seleção da questão de pesquisa; a segunda etapa, estabelecimento de critérios para 
inclusão e exclusão de estudos ou busca na literatura; a terceira etapa, definição das informações a serem extraídas dos estudos selecionados e a categorização dos estudos; quarta etapa, avaliação dos estudos incluídos; quinta etapa, interpretação dos resultados e a sexta etapa, apresentação da revisão/síntese do conhecimento.

A busca dos artigos foi realizada na biblioteca virtual sendo as bases de dados utilizadas: dados Literatura Latino-Americana e do Caribe em Ciências da Saúde (LILACS), ScientificElectronic Library Online (SciELO) e Google acadêmico. Optou-sepelas referidas biblioteca por disponibilizar periódicos brasileiros em textos completos e on-line.

Os critérios de inclusão na pesquisa serão: pesquisas que abordem as perspectivas dos graduandos em enfermagem quanto ao seu futuro profissional, publicadas em português; em formato de artigos, dissertações ou teses. Como critérios de exclusão: trabalhos que não apresentassem resumos na íntegra nas bases de dados e na biblioteca pesquisadas.

No processo de seleção dos artigos foram escolhidas os estudos que atendiam aos critérios. Nesse sentido, foram selecionados 11 artigos, após uma leitura flutuante dos trabalhos, buscando aproximações com a problemática a ser estudada. O método de escolha levou em consideração também o ano de publicação desses estudos, para se utilizar trabalhos com publicação mais recente, com pelo menos 5 anos de publicação.

\section{Resultados e Discussão}

Os 11 artigos selecionados foram publicados em periódicos diferentes. Ao analisa-los constatou-se que os artigos foram divulgados na: Revista Brasileira de Enfermagem com 05 artigos, Revista da Escola de Enfermagem da USP com dois artigos, Revista Mineira de Enfermagem com dois artigos, Escola Ana Nery (on line) com dois artigos, e os perídios Revista Enfermagem e Foco, Revista SANARE, Revista Gaúcha de Enfermagem, Revista Baiana de Saúde Pública e os Cadernos de Graduação: Ciênc. Biológicas e da Saúde, ambos com 1 artigo. Em relação ao ano de publicação, ocorre um predomínio de estudos no ano de 2014 com três estudos. (TABELA 1). 
Tabela 1. Caracterização dos artigos: Título, Autor, Ano de Publicação e Periódico.

\begin{tabular}{|c|c|c|c|}
\hline Título & Autor (es) & $\begin{array}{c}\text { Ano de } \\
\text { Publicação }\end{array}$ & Periódico \\
\hline $\begin{array}{l}\text { 1. Formação profissional e inserção no } \\
\text { mercado de trabalho: percepções de egressos } \\
\text { de graduação em enfermagem. }\end{array}$ & $\begin{array}{l}\text { COLENCI, } \\
\text { BERTI, H. W. }\end{array}$ & 2012 & $\begin{array}{lcl}\text { Revista } & \text { Esc. } & \text { de } \\
\text { Enfermagem da USP } & \end{array}$ \\
\hline $\begin{array}{l}\text { 2. Profissionais de Enfermagem habilitados } \\
\text { para o mercado de trabalho em Minas Gerais }\end{array}$ & $\begin{array}{l}\text { RIBEIRO, G. K. A; } \\
\text { et al. }\end{array}$ & 2014 & $\begin{array}{l}\text { Revista Mineira de } \\
\text { Enfermagem }\end{array}$ \\
\hline $\begin{array}{l}\text { 3. O inesperado futuro profissional: } \\
\text { Perspectiva do graduando de enfermagem }\end{array}$ & $\begin{array}{l}\text { OLIVEIRA, A. P. S.; } \\
\text { NUNES, S. L; } \\
\text { MUSSE, J. O. }\end{array}$ & 2016 & $\begin{array}{l}\text { Cadernos de Graduação: } \\
\text { Ciênc. Biológicas e da } \\
\text { Saúde }\end{array}$ \\
\hline $\begin{array}{l}\text { 4. Mercado de Trabalho da Enfermagem: } \\
\text { Aspectos Gerais }\end{array}$ & $\begin{array}{l}\text { MACHADO, M. H.; } \\
\text { et al. }\end{array}$ & 2016 & $\begin{array}{lll}\text { Revista } & \text { Enfermagem } & \mathrm{e} \\
\text { Foco } & & \\
\end{array}$ \\
\hline $\begin{array}{l}\text { 5. Inserção de egressos do curso de graduação } \\
\text { em Enfermagem no Mercado de Trabalho }\end{array}$ & $\begin{array}{l}\text { OLIVEIRA，F. M. } \\
\text { M.; et al }\end{array}$ & 2014 & Revista SANARE \\
\hline $\begin{array}{lll}\text { 6. Percepções de acadêmicos sobre } & \text { a } \\
\text { Enfermagem: } & \text { escolha, formação } & \text { e } \\
\text { competências da profissão } & & \\
\end{array}$ & $\begin{array}{l}\text { JABBUR, M. F. L. } \\
\text { O.; COSTA, S. M.; } \\
\text { DIAS, O. V. }\end{array}$ & 2012 & $\begin{array}{l}\text { Revista Mineira } \\
\text { Enfermagem }\end{array}$ \\
\hline $\begin{array}{l}\text { 7. Processo de formação e inserção no mercado } \\
\text { de trabalho: uma visão dos egressos de } \\
\text { Enfermagem. }\end{array}$ & $\begin{array}{l}\text { CANEVER, B. P.; et } \\
\text { al }\end{array}$ & 2014 & $\begin{array}{l}\text { Revista Gaúcha de } \\
\text { Enfermagem }\end{array}$ \\
\hline $\begin{array}{l}\text { 8. Inserção no mercado de trabalho: trajetória } \\
\text { de egressos de um curso de graduação em } \\
\text { Enfermagem }\end{array}$ & JESUS, B. H.; et al & 2013 & Escola Ana Nery (online) \\
\hline $\begin{array}{l}\text { 9. Egressas da Escola de Enfermagem Carlos } \\
\text { Chagas: campos de atuação. }\end{array}$ & $\begin{array}{l}\text { SANTOS, F. B. O.; } \\
\text { MARQUES, R. C. }\end{array}$ & 2015 & Escola Ana Nery (online) \\
\hline $\begin{array}{l}\text { 10. Expansão dos Cursos de Graduação em } \\
\text { Enfermagem: dilemas e contradições frente ao } \\
\text { mercado de trabalho }\end{array}$ & SILVA, K. L.; et al. & 2013 & $\begin{array}{l}\text { Revista } \quad \text { Esc. } \\
\text { Enfermagem da USP }\end{array}$ \\
\hline $\begin{array}{l}\text { 11. Formação Acadêmica e a Prática } \\
\text { Profissional de Enfermagem: Interfaces para } \\
\text { reflexão. }\end{array}$ & $\begin{array}{l}\text { TREVISAN, D. D.; } \\
\text { et al }\end{array}$ & 2014 & $\begin{array}{l}\text { Revista Baiana de Saúde } \\
\text { Pública }\end{array}$ \\
\hline
\end{tabular}

Fonte: Dados do Pesquisador

Numa análise do número de publicações, o SEMESP (2015) aponta que o aumento exponencial no número de IES, pode ser provocado pelo enorme número de egressos procurando o mercado de trabalho, aumentando a preocupação desta inserção.

$\mathrm{Na}$ análise da caracterização do perfil dos alunos, os artigos mostram em sua maioria que são mulheres, com média de 20 à 30 anos. Esse fato é verificado na maioria dos os estudos, como os de Colenci e Berti (2012) em que 85\% da amostra eram mulheres em sua maioria com 28 anos; Oliveira et al (2016) onde ocorre predominância do sexo feminino (88\%) na amostra, com média de idade entre 20 e 30 anos; Jabbur, Costa e Dias (2012), em que a maioria dos acadêmicos é do sexo feminino (70\%), com idade entre 17 e 23 anos; Canever et al (2014), onde se apresenta uma amostra com maioria de mulheres (90\%) e idades entre 22 e 31 anos. 
Em relação ao processo de formação acadêmica e expectativas profissionais, os estudos, em sua maioria, apontam um resultado similar, pois evidencia-se frente as amostras que os graduandos de enfermagem tem forte insegurança frente ao mercado de trabalho, principalmente aqueles em processo final de graduação.

No estudo de Silva et al (2013) aponta-se entre os alunos participantes da pesquisa um sentimento de insegurança quanto à questão da empregabilidade na enfermagem e aos problemas oriundo da formação acadêmica. Nesse sentido Machado et al (2016), enumeram que os problemas mais alegados, pelos alunos graduandos de enfermagem na pesquisa, para conseguir emprego são a falta de experiência profissional, além da formação acadêmica insuficiente, o que os leva a procurar imediatamente após a graduação, um curso de especialização.

No estudo de Colenci e Berti (2012), percebe-se que a insegurança, provém de uma formação acadêmica com dificuldades de desenvolver a aprendizagem com a prática, ou seja, ocorre uma incompatibilidade entre formação e prática profissional, devido a metodologias pedagógicas desvinculados da realidade do desenvolvimento do aluno. Também enumera-se a falta de conhecimento sobre gerência, estágios e carga horária insuficientes para o desenvolvimento de habilidades necessárias à assistência; além da falta de incentivos à pesquisa.

Jesus et al (2013), ao versar sobre a questão da insegurança, ressaltam que existe certa insegurança dos egressos em admitir as suas capacidades, além de muitos demonstrarem frustração em não conseguir um emprego por concurso, o que leva os mesmos a buscarem emprego por meio de currículos, onde se pesa a experiência profissional, que se torna uma dificuldade, pois, para a grande maioria das instituições de saúde, esse é um fator de grande importância para a contratação de um profissional.

Quanto a apresentação das principais competências e habilidades para o desenvolvimento profissional na área da saúde, os estudos mostram que a graduação em Enfermagem ainda não comtempla todas as necessidades, havendo um distanciamento da teoria e prática.

Segundo Trevisan et al (2014), a questão da insegurança, também esta presente na correlação entre conhecimentos teóricos e prática, sendo necessária uma reorganização do currículo às necessidades da área da saúde, para contornar demandas relacionadas as 
competências, habilidades, gestão e liderança, criatividade e posicionamento ético profissional, visto que as mesmas são essenciais para inserção no ambiente mercadológico.

Nessa mesma ótica Colenci e Berti, (2012) apontam que um dos fatores que mais contribuem para a dificuldade de inserção dos graduados de enfermagem no mercado é a falta de experiência. Numa análise similar, Jesus et al (2013), ao versar sobre a importância das competências e habilidades assimiladas no âmbito acadêmico, ressalta que devido o atual mercado de trabalho dispor de um número considerável de profissionais, as empresas acabam tendo o privilegio de escolher os mais capacitados e experientes, ou seja, opta-se pelos enfermeiros com melhor formação, qualificação e maior experiência na profissão

Oliveira et al (2014), em seus estudos apontam que de forma geral, os participantes apontaram que as competências adquiridas na graduação os prepararam parcialmente para exercer a profissão e não garantem experiências suficientes dentro daquela em variados setores da saúde.

Assim, como observado na maior parte dos estudos analisados, é necessário que os graduandos tenham a realização da prática de estudos, para que possam desenvolver as habilidades e competências realmente necessárias.

Isso nos mostra que em meio a tal dificuldade devem ser (re)analisado os currículos dos cursos de graduação em Enfermagem, para que as necessidades e questionamentos sejam revisados, possibilitando a realização de uma análise frente à possibilidade de adequação às necessidades solicitadas, , trazendo cada vez mais o acadêmico mais próximo da realidade a ser enfrentada.

Nessa lógica, conforme apontam Canever et al (2014) é necessário que se repense o processo de ensino e aprendizagem nos cursos de graduação em Enfermagem, pois a realidade e o cotidiano profissional dessa área demonstram que deve-se pensar em uma formação diferenciada que contribua para a inserção dos graduandos no mercado de trabalho.

Com semelhança na ideia acima, Paranhos e Mendes (2010, apud OLIVEIRA et al, 2016), ressaltam que o processo ensino e aprendizagem na enfermagem é fundamental para a formação de profissionais mais criativos e adaptativos, capazes de se adequar as realidades impostas nos serviços de saúde e, ao mesmo tempo, comprometidos com a vida.

Jesus et al (2013), ao versarem sobre essa questão, enunciam que o grande desafio dos docentes dos cursos de graduação em Enfermagem educadores está no processo de formação 
de profissionais aptos a responderem às demandas do mercado, nesse sentido, é importante que se realize diagnósticos sobre a situação dos formandos e do atual ambiente de trabalho do enfermeiro, para que se realize um olhar sobre esse contexto.

Para Ribeiro et al (2014), essa realidade apresentada, define a enfermagem no Brasil, pois existe a necessidade de se qualificar continuamente. Essa evidenciação leva a consolidar a ideia de melhoria do processo de formação acadêmica, pois as demandas e transformações do mundo da saúde são constantes, sendo necessário uma atualização constante do currículo do curso de graduação em Enfermagem para atender as demandas.

Dessa forma, como salientam Oliveira et al (2014), o processo de inserção do futuro Enfermeiro no mercado de trabalho, passa pelo "tripé" que relaciona a devida formação do estudante, as características pessoais (habilidades e competências assimiladas) e as atuais exigências da área da saúde, que dispõe de uma grande oferta de trabalhadores, e busca agregar somente aqueles com maior potencial e qualificação

Portanto, o grande desafio para a Enfermagem, segundo a análise dos artigos escolhidos para essa revisão, é o de formular proposições que impliquem na real melhoria da condição dos profissionais, seja no âmbito acadêmico ou no ambiente laboral. Assim, a principal responsabilidade é das instituições de ensino superior que oferecem a graduação em Enfermagem, sendo dever das mesmas formar os futuros profissionais da saúde, frente às reais necessidades do mercado de trabalho.

\section{Considerações Finais}

Mediante as conceptualizações presentes nesse estudo, em conjunto com a análise dos artigos selecionados, pode-se perceber que o mercado de trabalho da área da saúde está em constante transformação, sendo necessário ao Enfermeiro que queira se inserir na mesma uma modelagem e adequação as necessidades exigidas pelas empresas.

Segundo os estudos, percebe-se que os formandos em Enfermagem, devem se aprimorar na atividade profissional a ser executada, através da aquisição e assimilação de habilidades e competências, para que possam desenvolver com qualidade e eficiência a promoção da saúde. 
No entanto, enumera-se frente aos resultados analisados, que a maior parte das pesquisam enunciam dificuldades de inserção dos Enfermeiros recém formados, visto que cobra-se experiência e conhecimentos que nem sempre são conseguidos no âmbito acadêmico, sendo necessário um processo de práticas que a maioria das instituições de ensino superior não possibilitam.

Dessa forma, verifica-se nesse estudo, que existe uma grande lacuna no processo de ensino e aprendizagem nos cursos de graduação em Enfermagem, pois todo o aprendizado teórico não consegue se converter em práticas de saúde devido a falta de um currículo voltado ao atendimento das demandas do mercado de trabalho, que necessitam de um profissional preparado para atuar em diversas áreas e dominante de habilidades e competências necessárias para a realização de seu trabalho

Portanto, ressalta-se aqui a importância e necessidade de melhoria da qualificação do graduando em Enfermagem, para que o mesmo possa desenvolver-se profissionalmente, de modo crítico e reflexivo, atendendo todas as necessidades do mercado de trabalho.

\section{Referências}

BARLEM, J. G. T.; et al. Fragilidades, fortalezas e desafios na formação do enfermeiro. Escola Anna Nery [online], v.16, n.2, Rio de Janeiro, abr-jun. 2012.

CANEVER, B.P. et al. Processo de Formação e Inserção no Mercado de Trabalho: Uma Visão dos Egressos de Enfermagem. Rev. Gaúcha Enfermagem, vol.35, n.1, 2014.

COLENCI, R.; BERTI, H. W. Formação profissional e inserção no mercado de trabalho: percepções de egressos de graduação em enfermagem. Revista Esc. de Enfermagem da USP, Botucatu-SP, 2012.

IBGE. Infográficos: dados gerais do município. Disponível em: <http://cidades .ibge.gov.br/xtras/perfil.php?codmun=270630>. Acesso em 19 de março de 2017.

ITO, E. E.et al . O ensino de enfermagem e as diretrizes curriculares nacionais: utopia $\mathrm{x}$ realidade. Revista Esc. de Enfermagem da USP, São Paulo, v. 40, n. 4, Dez. 2006.

JABBUR, M. F. L. O.; COSTA, S. M.; DIAS, O. V. Percepções de acadêmicos sobre a Enfermagem: Escolha, Formação e Competências da Profissão. Revista Norte Minas Enfermagem, v.1, n.1, p.3-16. Montes Claros - Minas Gerais, 2012.

JESUS, B.H. et al. Inserção no mercado de trabalho: trajetória de egressos de um curso de graduação em enfermagem. Esc. Anna Nery, [online], Rio de Janeiro, v. 17, n. 2, Junho, 2013. 
KRAEMER, F. Z.; DUARTE, M. L. C.; KAISER, D. E. Autonomia e trabalho do enfermeiro. Rev. Gaúcha Enferm., Porto Alegre, v. 32, n. 3, Set. 2011.

MACHADO, M. H.; et al. Mercado de Trabalho da Enfermagem: Aspectos Gerais. Revista Enfermagem e Foco, 2015.

MENDES, K. D. D, SILVEIRA, R. C. C. P.; GALVÃO, C. M. Revisão integrativa: método de pesquisa para a incorporação de evidências na saúde e na enfermagem. Texto \& contexto enfermagem, 2008.

OLIVEIRA, A. P. S.; NUNES, S. L.; MUSSE, J. O. O Inesperado Futuro Profissional: Perspectiva do Graduando de Enfermagem. Cadernos de Graduação de Ciências Biológicas e de Saúde Unit, v.3, n.3, Aracaju - Sergipe, 2016.

OLIVEIRA, F. M. M.; et al. Inserção de Egressos do Curso de Graduação em Enfermagem no Mercado de Trabalho. Revista SANARE, Sobral, V.13, n.1, 2014.

REBOLCAS, L. C.; FERNANDES, J. D. Uma década de Diretrizes Curriculares Nacionais para a graduação em Enfermagem: avanços e desafios. Revista Brasileira de Enfermagem, v. 66, Brasília, SEPT. 2013.

RIBEIRO, G. K. A; et al. Profissionais de Enfermagem habilitados para o mercado de trabalho em Minas Gerais. Revista Mineira de Enfermagem, Belo Horizonte-MG, 2014.

SANTOS, F.B.O.; MARQUES, R.C. Egressas da Escola de Enfermagem Carlos Chagas: campos de atuação. 1936-1948. Esc. Anna Nery [online], vol.19, n.2, 2015.

SILVA, D.G.V. et al. Os desafios enfrentados pelos iniciantes na prática de enfermagem. Revista Escola de Enfermagem da USP, São Paulo, v.44, n.2, p.511-516, 2010.

SILVA, L.K. et al. Expansão dos Cursos de Graduação em Enfermagem: dilemas e contradições frente ao mercado de trabalho. Revista Escola de Enfermagem da USP, vol.47, no.5, Outubro, 2015.

TREVISAN, D.D. et al. Formação Acadêmica e a Prática Profissional de Enfermagem: Interfaces para reflexão. Rev. Baiana de Saúde Pública, v.38, n.1, jan./mar. 2014.

Como citar este artigo (Formato ABNT):

NASCIMENTO, Patrícia N.; BARBOSA, Mirella C.L. Perspectivas dos Graduandos de Enfermagem Frente ao Mercado de Trabalho. Id on Line Revista Multidisciplinar e de Psicologia, Maio de 2017, vol.11, n.35, p.267278. ISSN: 1981-1179.

Recebido: 19.02.2017

Aceito: .08.04.2017 